\title{
Mô phỏng ứng xử uốn tấm sàn bê tông cốt thép sử dụng mô hình vật liệu coupled damage-plasticity microplane (CDPM)
}

\author{
Nguyễn Thái Bình ${ }^{1}$, Võ Khắc Lê Anh ${ }^{1}$, Lương Văn Hải ${ }^{1}$, Cao Nguyên Thi ${ }^{2}$ \\ ${ }^{1}$ Khoa Kỹ Thuật Xây Dựng, Trường Đại học Bách khoa TP HCM, Đại học Quốc Gia TP HCM \\ ${ }^{2}$ Bộ môn Kỹ thuật Xây dựng, Khoa Kỹ thuật Công nghệ, Trường Đại học Tiền Giang
}

\begin{tabular}{|c|c|}
\hline Từ KHOÁ & TÓM TẮT \\
\hline Mô hình vật liệu Coupled & Bài báo tập trung vào việc áp dụng mô hình vật liệu Coupled Damage-Plasticity Microplane (CDPM) được \\
\hline Damage-Plasticity Microplane & trang bị trên phần mềm ANSYS trong mô phỏng ứng xử phi tuyến của vật liệu bê tông. Đặc tính phi tuyến \\
\hline$(\mathrm{CDPM})$ & của tấm sàn bê tông cốt thép (BTCT) chịu uốn thiết kế theo tiêu chuẩn ACI 318-08 gia tải tĩnh được phân \\
\hline $\begin{array}{l}\text { Ửng xử phi tuyến vật liệu bê } \\
\text { tông }\end{array}$ & $\begin{array}{l}\text { tích dựa trên phương pháp phần tử hữu hạn (PTHH). Mô hình PTHH ba chiều của thí nghiệm uốn ba } \\
\text { điềm tâm sàn BTCT được xây dưng trong phần mềm ANSYS/Workbench (ANSYS Student 2020). Các phần }\end{array}$ \\
\hline Phần tử cpt215 & tử khối tám nút CPT215 tích hộp mô hình CPDM được sử dụng trong mô phỏng cho vật liệu bê tông trong \\
\hline Phần tử reinf264 & $\begin{array}{l}\text { khi các phần tử nhúng REINF264 được dùng để mô phỏng sự làm việc của cốt thép. Một phần quan trọng } \\
\text { của bài báo này là việc tồng hợp cách xác định các thông số đầu vào của mô hình CDPM (15 thông số) } \\
\text { phù hợp với tấm sàn BTCT chịu uốn. Kết quả mô phỏng số của biểu đồ quan hệ tải trọng-chuyển vị tại } \\
\text { điểm giữa tấm sàn BTCT được trình bày và so sánh với kết quả thí nghiêm của nghiên cứu trước đây. }\end{array}$ \\
\hline
\end{tabular}

\section{KEYWORDS}

Coupled Damage-Plasticity

Microplane model

Nonlinear behavior of concrete

CPT215 element

REINF264 element

\begin{abstract}
The article focuses on the application of the Coupled Damage-Plasticity Microplane (CDPM) material model equipped with ANSYS software in simulating the nonlinear behavior of concrete materials. The nonlinear behavior of the reinforced concrete (RC) slab which was designed according to ACI 318-08 standard under monotonically increasing load is analyzed based on The Finite Element Method. The threedimensional finite element model of the three-point bending test of the RC slab was built in ANSYS/Workbench software (ANSYS Student 2020). The solid eighth-node CPT215 elements are employed in the simulation for concrete material whereas the embedded REINF264 elements are used to simulate the rebars. A crucial part of this paper is a determination of the suitable input parameters of the CDPM material model (15 parameters) for the considered flexural RC slab. The numerical results of the relationship between the applied load and the displacement at the mid-span of the RC slab are presented and compared with the experimental results of the previous study.
\end{abstract}

\section{Giới thiệu:}

Việc mô phỏng và phân tích phần tử hữu hạn (PTHH) cho các kết cấu bê tông cốt thép như dầm, cột và sàn hiện nay vẫn là một thách thức lớn cho các nhà nghiên cứu vì bê tông là vật liệu không đồng nhất với các ứng xử cơ học phi tuyến phức tạp [1]. Đã có rất nhiều mô hình vật liệu được đề xuất cho bê tông từ các nghiên cứu trước đây [2-3]. Mỗi mô hình vật liệu đều có điểm mạnh và điểm yếu khác nhau, do đó việc lựa chọn một mô hình vật liệu có tính thuyết phục cao để ứng dụng tính toán có hiệu quả cho vật liệu bê tông hiện vẫn đang là một vấn đề mang tính thời sự [4]. Mô hình liên tục thường hay được sử dụng trong mô phỏng số vật liệu bê tông, trong đó mối quan hệ ứng suất - biến dạng thường được sử dụng để diễn tả khả năng chịu kéo, nén là thông số đầu vào quan trọng. Các mô hình liên tục này thường được xây dựng trên mối quan hệ bậc cao giữa biến dạng và ứng suất. Mặc dù mô hình liên tục mô phỏng rất tốt đối với vật liệu đàn hồi tuyến tính, tuy nhiên khả năng mô phỏng ứng xử phi tuyến cho vật liệu bê tông của các mô hình liên tục này là rất giới hạn.
Ngược lại, mô hình vật liệu vi mô dựa trên cơ học vi mô (MicroMechanics) sẽ sử dụng một cách tiếp cận khác, đó là phát triển mối quan hệ ứng suất - biến dạng vĩ mô từ cơ chế của cấu trúc vi mô [5-9]. Mô hình CDPM hiện tại do phần mềm ANSYS trang bị trong thư viện vật liệu dựa trên nghiên cứu của Zreid và Kaliske [10-12]. Sự cải tiến này là một phát triển rất hữu ích do số lượng thông số phụ thuộc vào vật liệu giảm đáng kể từ 33 thông số đầu vào ở các phiên bản Microplane trước do Nguyen và cộng sự [13] đề xuất xuống còn 15 thông số như phiên bản hiện tại do Zreid và Kaliske [12] đề xuất. Một trong những loại phần tử phù hợp với mô hình vật liệu CDPM đáng chú ý là phần tử khối tám nút CPT215 sẽ là phần tử tiềm năng trong mô phỏng ứng xử phi tuyến của vật liệu bê tông.

Trong bài báo này, mô hình PTHH ba chiều của thí nghiệm uốn ba điểm tấm sàn BTCT được xây dựng bằng phần mềm ANSYS/Workbench, các phần tử khối CPT215 sử dụng mô hình vật liệu CPDM được sử dụng trong mô phỏng ứng xử phi tuyến cho phần vật liệu bê tông trong khi các phần tử nhúng REINF264 được sử dụng để mô phỏng ứng xử của các thanh cốt thép trong tấm sàn BTCT khảo sát. 
Việc xác định các thông số đầu vào trong mô hình CDPM cho tấm sàn BTCT được tổng hợp và đề xuất dựa trên việc khảo sát các tài liệu nghiên cứu liên quan. Kết quả mô phỏng số về quan hệ giữa tải trọng tác dụng tĩnh và chuyển vị tại điểm giữa tấm sàn được trình bày và so sánh với kết quả thí nghiệm của nghiên cứu trước đây do Cao và cộng sự (2015) [14] thực hiện. Sự tương đồng tốt giữa kết quả mô phỏng và thí nghiệm thể hiện tính hợp lý của các thông số cho mô hình vật liệu CDPM cũng như tính khả thi của mô hình PTHH đề xuất cho tấm sàn BTCT khảo sát.

\section{Mô hình mô phỏng thí nghiệm uốn 3 điểm của tấm sàn BTCT:}

\subsection{Sơ lượt về thí nghiệm uốn 3 điểm:}

Tấm sàn bê tông cốt thép được thiết kế theo tiêu chuẩn ACI 31808 [15] và được chế tạo trong phòng thí nghiệm bởi Cao và cộng sự [14]. Tấm sàn có kích thước $1000 \times 900 \times 150 \mathrm{~mm}$ và được gia cường các thanh cốt thép đường kính $10 \mathrm{~mm}$ có thiết kế như mô tả trong Hình 1. Lớp bê tông bảo vệ có chiều dày $20 \mathrm{~mm}$. Tấm sàn được thiết kế với cường độ bê tông là $35 \mathrm{MPa}$. Mẫu thí nghiệm được chế tạo và dưỡng hộ trong 28 ngày trước khi thí nghiệm gia tải kiểm tra khả năng chịu uốn.
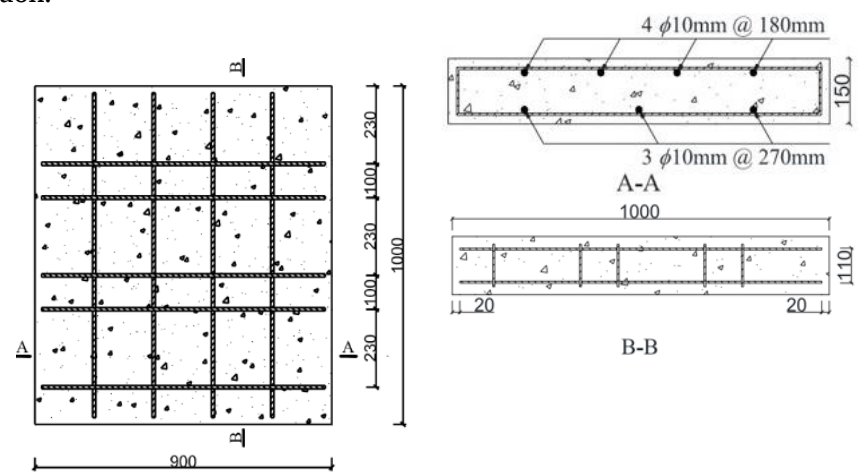

B-B

Hình 1. Thiết kế của tấm sàn BTCT trong thí nghiệm uốn 3 điểm [14].

\subsection{Mô hình vật liệu:}

\subsubsection{Vật liệu bê tông}

Các đặc tính cơ học của vật liệu bê tông được mô hình hóa thông qua mô hình vật liệu CDPM được trang bị trong phần mềm ANSYS. Mô hình CDPM này dựa trên nghiên cứu của Zreid và Kaliske [10 - 12]. Tuy nhiên, mô hình này chỉ có thể truy cập thông qua các dòng lệnh và không hiển thị trong giao diện của ANSYS/Workbench. Mô hình CDPM yêu cầu 15 thông số đầu vào [16], cách xác định các thông số này trong mô phỏng phi tuyến ứng xử của vật liệu bê tông được tổng hợp như sau:

Các thông số trong giai đoạn đàn hồi (Elasticity): $E$ và $v$ có thế được xác định từ vùng đàn hồi của đường cong ứng suất-biến dạng vật liệu, hoặc bằng cách sử dụng các công thức thí nghiệm có sẵn trong tài liệu [16]. Trong nghiên cứu này, hệ số Poisson là $v=0,2$ và mô đun đàn hồi $\left(E_{c}\right)$ cho bê tông được tính theo tiêu chuẩn ACI 318-08 [15] với công thức:

$$
E_{c}=4700 \sqrt{f_{u c}}(\mathrm{MPa})
$$

trong đó $f_{u c}$ là ứng suất lớn nhất, và biến dạng tương ứng $\varepsilon_{c}$ có thể được tính như công thức (2) được đề xuất bởi Feenstra và cộng sự [17] như sau:

$$
\varepsilon_{c}=\frac{4}{3} \frac{f_{u c}}{E_{c}}
$$

Thông số trong giai đoạn chảy dẻo (Plastictiy): các thông số độ bền $f_{u c}$, $f_{b c}$ và $f_{u t}$ là các thuộc tính cơ học của vật liệu phổ biến (có thể được tìm thấy bằng thí nghiệm). Trong trường hợp không có dữ liệu thí nghiệm đầy đủ, nếu biết $f_{u c}$ có thể sử dụng quan hệ sau $[16,18]$ :

$$
\begin{gathered}
f_{b c}=1,15 \cdot f_{u c}(\mathrm{MPa}) \\
f_{u t}=1,4 \cdot\left(\frac{f_{u c}}{10}\right)^{\frac{2}{3}}(\mathrm{MPa})
\end{gathered}
$$

Trong nghiên cứu này, tấm sàn được chế tạo bằng bê tông trộn sẵn với cường độ bê tông là $35 \mathrm{MPa}$. Cường độ chịu nén thu được từ các thí nghiệm được thực hiện trên các mẫu trụ tiêu chuẩn $(150 \times 300$ $\mathrm{mm}$ ) là 35,4 MPa do Cao và cộng sự thực hiện [14]. Do đó, cường độ chịu nén đơn trục $f_{u c}=35,4 \mathrm{MPa}$ cho tấm sàn BTCT khảo sát.

Việc xác định giá trị của thông số $\sigma_{v}^{c}$ tương đối phức tạp. Nếu thiếu thông tin để xác định, thông số này có thể được ước tính theo công thức kinh nghiệm được đề xuất trong tài liệu [16]:

$$
\sigma_{v}^{c}=-\frac{2}{3} f_{b c}(\mathrm{MPa})
$$

Thông số $R$ được mô tả chi tiết trong [12]. Thông thường, $R=2$.

Thông số hardening có thể được tính theo công thức (6) do Nguyen và Houlsby [19] đề xuất như sau:

$$
f_{c 0}=\frac{f_{u c} \cdot\left(E_{c}+D\right)}{E_{c}}-D \cdot \varepsilon_{c}(\mathrm{MPa})
$$

trong đó $f_{c 0}$ có giá trị khoảng $30 \%$ của ứng suất nén cực đại $f_{u c} ; D$ là thông số hardening; $E_{c}$ và $\varepsilon_{c}$ lần lượt là mô đun đàn hồi và biến dạng tương ứng tại điểm có giá trị ứng suất nén cực đại và được tính toán bằng công thức (1) và (2).

Thông số phá hoại (Damage parameters): các thông số $R_{T}, \beta_{t}$ và $\gamma_{t 0}$ được xác định từ thí nghiệm kéo lặp đơn trục. Trong trường hợp không có các dữ liệu từ thì nghiệm này, có thể lấy $R_{T}=1$, $\beta_{t}=1,5 \beta_{c}$ làm điểm bắt đầu. Ngưỡng bắt đầu xuất hiện phá hoại kéo $\gamma_{t 0}$ được lấy bằng không $\left(\gamma_{t 0}=0\right)$ vì sự phá hoại kéo gần như bắt đầu ngay lập tức ngay sau giới hạn đàn hồi [16]. Giá trị của các thông số phá hoại được xác định dựa trên đề xuất của Zreid và Kaliske [12] và được xác định để phù hợp với đường cong quan hệ tải trọng-chuyển vị bằng thí nghiệm.

Thông số nonlocal (Nonlocal parameters): thông số $m$ được lấy sao cho $m>1$, thông thường sẽ được lấy $m=2,5$ nhằm điều chỉnh giải pháp tính toán. Giá trị của thông số $c$ được hiểu là kích thước của vùng chịu thiệt hại của kết cấu, có thể tính toán bằng các xác định chiều dài đặc trưng của vật liệu theo nhiều cách khác nhau được đề xuất trong tài liệu [12]. 
Bảng 1. Thông số mô phỏng cho mô hình CDPM của vật liệu bê tông [5].

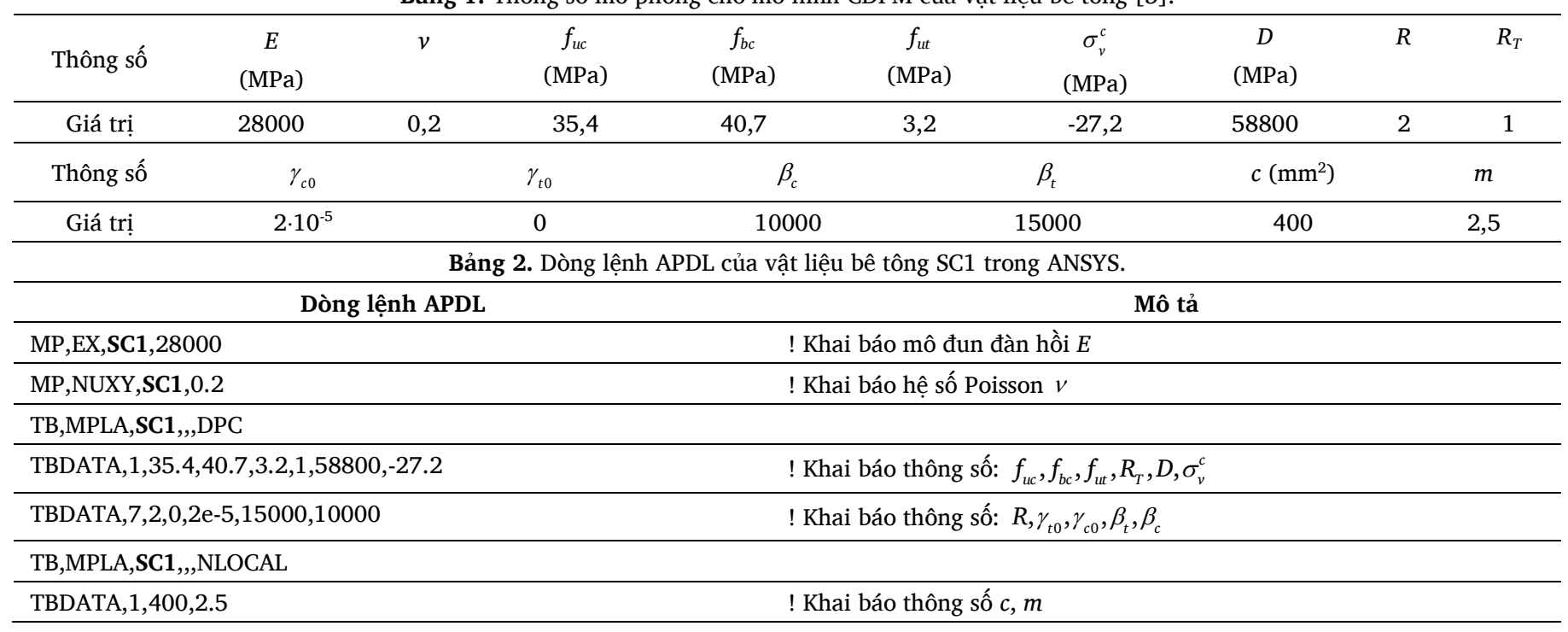

Các thông số vật liệu cho vật liệu bê tông trong mô phỏng được thể hiện trong Bảng 1. trong đó giá trị $f_{u c}=35,4 \mathrm{MPa}$ lây theo thí nghiệm của Cao và cộng sự [14]. Các dòng lệnh command APDL cho vật liệu bê tông trong ANSYS/Workbench cũng được trình bày trong Bảng 2.

\subsubsection{Cốt thép gia cường}

Trong thí nghiệm uốn 3 điểm, Cao và cộng sự [14] sử dụng cốt thép SD40 có đường kính $d=10 \mathrm{~mm}$, giới hạn chảy và cường độ kéo lần lượt là 390 và $560 \mathrm{MPa}$. Trong nghiên cứu này, các thanh thép trong mô hình PTHH được mô hình hóa với mối quan hệ ứng suất-biến dạng song tuyến như thể hiện trong Hình 2. Giới hạn chảy được xác định là $390 \mathrm{MPa}$ từ thí nghiệm, mô đun đàn hồi của thép $E_{s}=200 \mathrm{GPa}$, hệ số Poisson của các thanh thép được lấy bằng $v=0,3$ và mô đun tiếp tuyến của phần sau khi chảy dẻo được lấy là $E_{T}=0,005 \cdot E_{s}=1000 \mathrm{MPa}$ $[20]$.

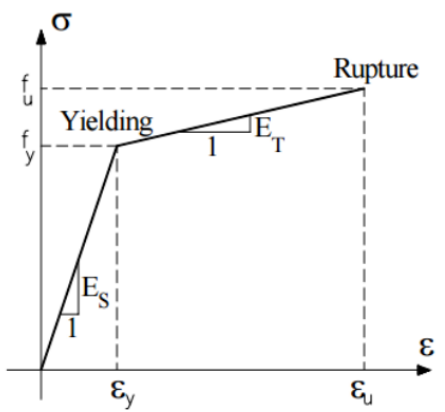

Hình 2. Quan hệ ứng suất biến dạng trong cốt thép [20].

\subsection{Thiết lập mô hình PTHH:}

Mô hình PTHH ba chiều của thí nghiệm uốn ba điểm cho tấm sàn BTCT được xây dựng trong phần mềm ANSYS/Workbench. Các phần tử khối tám nút CPT215 được sử dụng để mô phỏng ứng xử phi tuyến của phần vật liệu bê tông bằng mô hình $\mathrm{CDPM}$ trong khi các phần tử nhúng REINF264 được sử dụng để mô phỏng ứng xử của các thanh thép gia cường. Các phần sau sẽ trình bày chi tiết về phần tử khối CPT215, phần tử nhúng REINF264, mô hình mô phỏng, các kích thước lưới phần tử, và các điều kiện biên của mô hình PTHH đề xuất.

\subsubsection{Phần tử khối CPT215}

Mô hình vật liệu CDPM được trang bị với các phần tử khối (3-D) CPT214, СРT215, СРТ216 cũng như cho các phần tử phẳng (2-D) CPT211, СРT212, СРT213 [10]. Trong nghiên cứu này, phần tử khối tám nút CPT215 (Hình 3) được sử dụng để mô hình phần vật liệu bê tông của tấm sàn BTCT trong phần mềm ANSYS/Workbench.

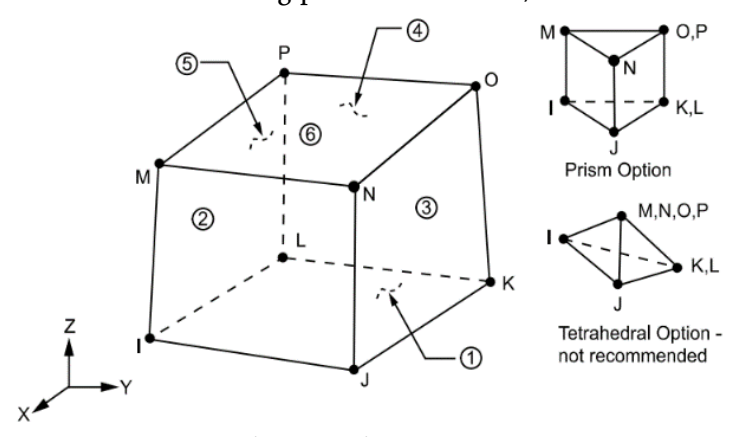

Hình 3. Phần tử khối 8 nút CPT215 [21].

\subsubsection{Phần tử nhúng REINF264}

Phần tử nhúng REINF264 là phần tử liên kết, gia cường thường được kết hợp với các phần tử khác (đặt vào bên trong các phần tử này) như phần tử dầm, phần tử tấm và phần tử khối,... (được gọi là phần tử cơ sở). Các vị trí nút, bậc tự do của phần tử nhúng REINF264 sẽ biến đổi một cách tự động để giống hoàn toàn với các phần tử cơ sở của nó, điều này là một đặc điểm đặc biệt của loại phần tử nhúng REINF264, làm cho việc sử dụng phần tử này vào mô hình sẽ dễ dàng hơn [21]. Trong nghiên cứu này, phần tử nhúng REINF264 được sử dụng cùng với phần tử khối tám nút CPT215 để mô phỏng ứng xử của thanh cốt thép cho tấm sàn BTCT và được thể hiện trong Hình 4. 


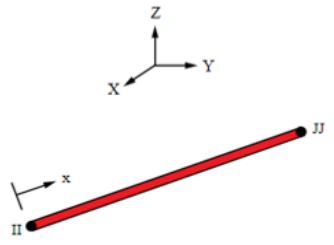

(a)

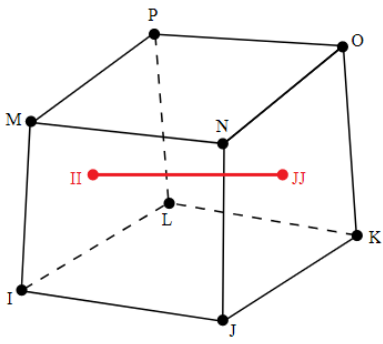

(b)

Hình 4. (a) Phần tử nhúng REINF264; (b) Phần tử nhúng REINF264 và phần tử cơ sở CPT215 [21].

\subsubsection{Kích thước và các điều kiện biên}

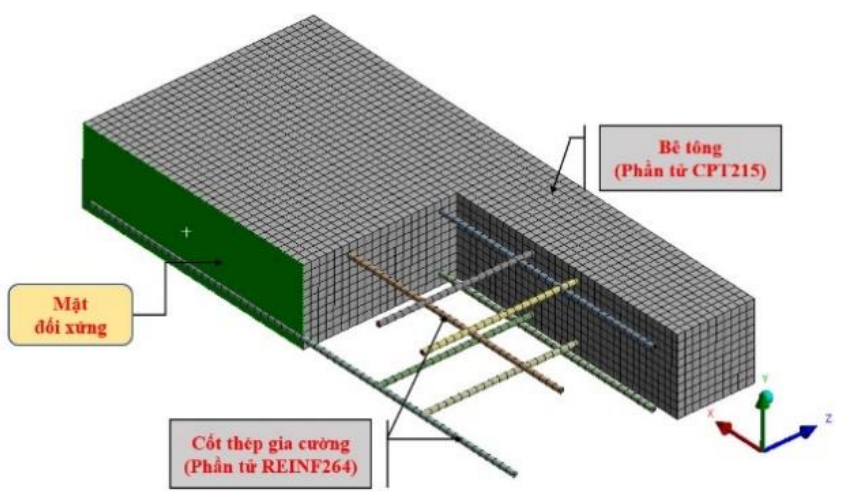

(a)

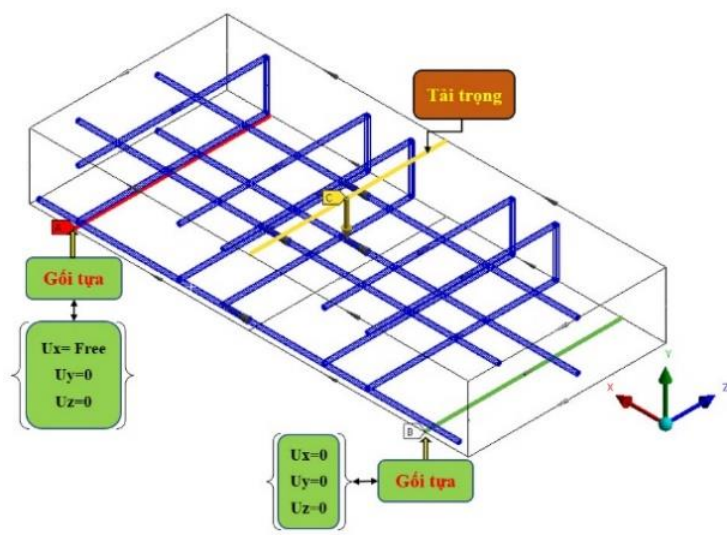

(b)

Hình 5. (a) Mô hình mô phỏng ba chiều của một nửa tấm sàn BTCT khảo sát; (b) Các điều kiện biên.

Mô hình PTHH cho tấm sàn BTCT được mô phỏng với các kích thước, thiết kế cốt thép, điều kiện biên động học và điều kiện biên tĩnh học được dựa trên mẫu thí nghiệm đối chứng của Cao và cộng sự [14]. Để giảm khối lượng tính toán, do tính chất đối xứng 1 trục của mẫu khảo sát, một nửa tấm sàn BTCT được mô phỏng với mặt đối xứng thể hiện như trong Hình 5(a). Các điều kiện biên tĩnh học và động học được thể hiện trên Hình 5(b). Trong mô hình này, tải trọng sẽ được áp dụng lên mô hình theo hình thức độ dịch chuyển thẳng đứng hướng xuống (theo phương $\mathrm{Y}$ ) ở vị trí giữa nhịp của tấm, và giá trị tối đa bằng $8 \mathrm{~mm}$ ứng với giá trị tối đa được thực hiện trong thí nghiệm.

Để khảo sát sự hội tụ của nghiệm số cũng như độ nhạy của kết quả nhận được từ mô phỏng, mô hình được khảo sát bằng các loại lưới phần tử là các khối hình lập phương. Do sự hạn chế về số phần tử đối với bản quyền ANSYS Student 2020, do đó 3 lưới phần tử có kích thước từ $40 \mathrm{~mm}, 30 \mathrm{~mm}$ và $20 \mathrm{~mm}$ tương ứng với các lưới chia Mesh 1 , Mesh 2 và Mesh 3 được khảo sát.

\section{Kết quả mô phỏng số:}

Kết quả mô phỏng số về quan hệ giữa tải trọng-chuyển vị tại vị trí giữa nhịp của tấm sàn BTCT qua các kích thước lưới khác nhau được trình bày và so sánh với dữ liệu thí nghiệm được thể hiện trong Hình 6. Có thể thấy rằng kết quả mô phỏng số là hội tụ thể hiện ứng xử tương tự với kết quả từ thí nghiệm đối chứng của Cao và cộng sự [14].

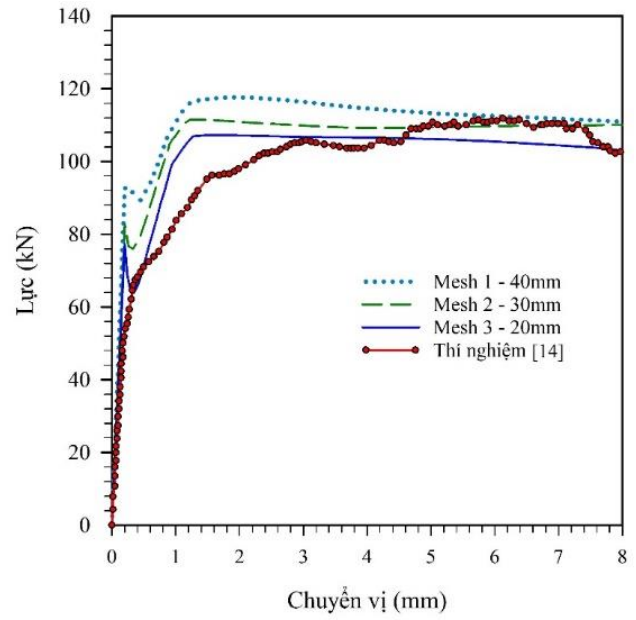

Hình 6. So sánh kết quả lực-chuyển vị giữa nhịp của tấm sàn BTCT giữa mô phỏng và thí nghiệm đối chứng.

Từ nghiên cứu hội tụ, có thể nhận thấy rằng mô hình PTHH được đề xuất mang lại các giải pháp số ổn định và hầu như không có sự khác biệt đáng chú ý giữa các kết quả từ các lưới chia phần tử khác nhau ở giai đoạn đầu (nhánh tăng). Ưu điểm của mô hình PTHH đề xuất là mô tả được cả ứng xử giai đoạn sau (nhánh giảm) của tấm sàn BTCT khảo sát đến hết giai đoạn thí nghiệm (chuyển vị tối đa là $8 \mathrm{~mm}$ trong thí nghiệm [14]).

Tải trọng cực hạn mà tấm sàn BTCT chịu được của cả ba trường hợp kích thước lưới phần tử khác nhau cùng với kết quả thí nghiệm [14] được thể hiện trong Bảng 3, trong đó số trong ngoặc đơn thể hiện sai số \% giữa kết quả mô phỏng và thí nghiệm. Có thể thấy rằng, giá trị mô phỏng PTHH khá phù hợp với kết quả của thí nghiệm đối chứng. Giá trị mô phỏng số tải trọng cực hạn của lưới phần tử khảo sát mịn nhất (Mesh $3-20 \mathrm{~mm}$ ) có sai khác với giá trị thí nghiệm vào khoảng $4 \%$ trong nghiên cứu này.

Sự sai khác nhỏ giữa kết quả mô phỏng và kết quả thí nghiệm đối chứng có thể lý giải là do chưa có số liệu thí nghiệm cụ thể về hệ số biến dạng $\gamma_{c 0}$ tương ứng với ngưỡng bắt đầu phá hoại do nén của bê tông trong mô hình vật liệu CPDM. Trong nghiên cứu mô phỏng này, giá trị này được tham số hóa theo đề xuất trong nghiên cứu của Pirker [9] và kết quả số trình bày là lời giải hội tụ tốt nhận được. 
Bảng 3. So sánh tải trọng cực đại ứng với các kích thước lưới chia khác nhau.

\begin{tabular}{ccccc}
\hline Mẫu SC1 & $\begin{array}{c}\text { Thí nghiệm } \\
\text { [5] }\end{array}$ & Mesh 1 & Mesh 2 & Mesh 3 \\
\hline Tải trọng & & 117,66 & 111,60 & $\begin{array}{c}107,21 \\
\text { cực đại } \\
(\mathbf{k N})\end{array}$ \\
\hline
\end{tabular}

(a)

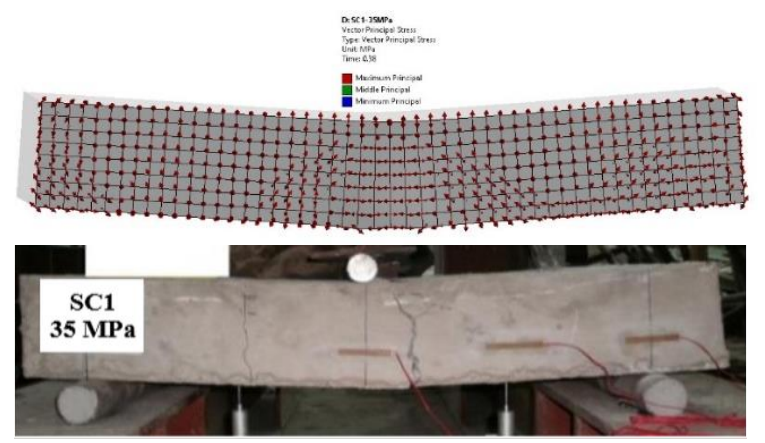

Hình 7. So sánh vùng xuất hiện vết nứt trong tấm sàn BTCT giữa (a) mô hình và (b) thí nghiệm.

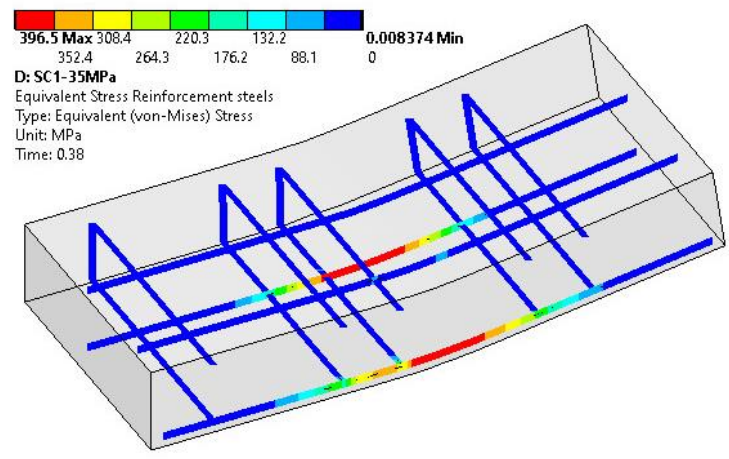

(a)

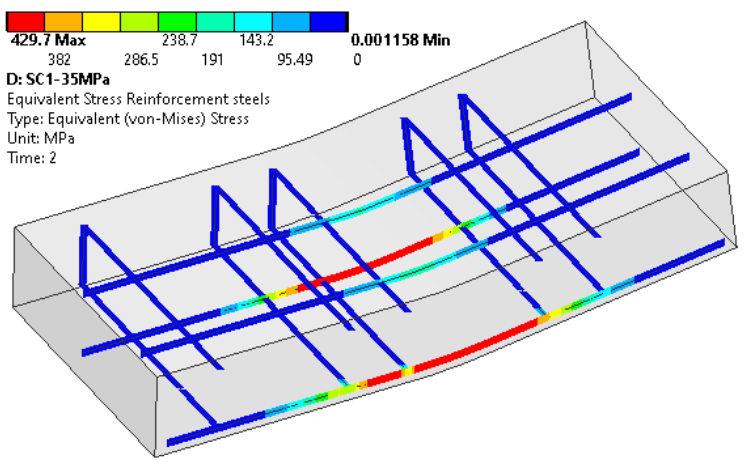

(b)

Hình 8. Ứng suất trong cốt thép gia cường của tấm sàn BTCT: (a) tại thời điểm tấm BTCT chịu lực cực hạn; (b) tại thời điểm ứng với chuyển vị giữa tấm sàn là $8 \mathrm{~mm}$.

Các vectơ ứng suất chính trong tấm sàn BTCT chịu lực tại thời điểm ứng với tải trọng cực hạn được thể hiện trên Hình $7(\mathrm{a})$. Từ phương của các ứng suất chính cho thấy có sự tương đồng của vùng xuất hiện ứng suất chính trên mô hình và vùng xuất hiện các vết nứt nhỏ được quan sát thấy trên mẫu thí nghiệm thể hiện trên Hình 7(b) ứng với thời điểm tải trọng cực hạn tương ứng. Mặc dù mô hình PTHH được đề xuất chưa mô tả chính xác vị trí các vết nứt, nhưng có thể chỉ ra được vùng nứt tương tự như quan sát trong thí nghiệm.

Ứng suất trong cốt thép gia cường tại thời điểm tấm sàn chịu lực cực hạn và tại thời điểm ứng với chuyển vị tối đa thực hiện trong thí nghiệm (8 mm) được thể hiện trên Hình 8 . Kết quả mô phỏng cho thấy cốt thép gia cường làm việc trong giai đoạn chảy dẻo trong cả 2 thời điểm khảo sát, thể hiện sự hợp lý của hàm lượng cốt thép gia cường đã sử dụng của tấm sàn BTCT khảo sát.

\section{Kết luận:}

Mô hình PTHH có khả năng mô phỏng ứng xử uốn của tấm sàn BTCT đã được xây dựng thành công bằng phần mềm ANSYS/Workbench. Trong mô hình PTHH được đề xuất, các phần tử khối CPT215 tích hợp mô hình vật liệu CDPM được sử dụng để mô phỏng ứng xử phi tuyến của vật liệu bê tông trong khi các phần tử nhúng REINF264 được sử dụng để mô phỏng ứng xử của các thanh cốt thép. Kết quả mô phỏng số tương đồng với kết quả thí nghiệm đối chứng.

Các kết quả số từ mô hình khá ổn định qua cả 3 kích thước lưới khảo sát. Sự thành công của mô hình PTHH đề xuất này phải kể đến sự đóng góp to lớn của mô hình vật liệu CDPM được trang bị cho phần tử CPT215 để mô tả ứng xử phi tuyến của vật liệu bê tông. Các thông số mô hình vật liệu CDPM được đề xuất cho tấm sàn BTCT khảo sát là tương đối phù hợp với nghiên cứu thí nghiệm đối chứng.

Với kết quả tích cực thu được từ mô phỏng trong nghiên cứu này, mô hình PTHH được đề xuất hứa hẹn sẽ hiệu quả trong việc phân tích ứng xử phi tuyến của các tấm BTCT chịu uốn trong các tình huống phức tạp hơn. Ví dụ, các tấm BTCT được sửa chữa/gia cố bằng vật liệu FRP (Fiber Reinforced Polymer) bằng cách sử dụng kỹ thuật gia cường bên ngoài (EBR-Externally Bonded Reinforcement) hoặc gia cường gần bề mặt (NSM-Near-Surface Mounted).

\section{Lời cảm ơn}

Nghiên cứu này được tài trợ bởi Trường Đại học Bách Khoa Tp.HCM trong khuôn khổ đề tài Khoa học Công nghệ cấp trường năm 2020, mã số: T-KTXD-2020-23. Chúng tôi xin cảm ơn Trường Đại học Bách Khoa, ĐHQG-HCM đã hỗ trợ thời gian, phương tiện và cơ sở vật chất cho nghiên cứu này./.

\section{Tài liệu tham khảo}

[1] Grassl, P., Xenos, D., Nyström, U., Rempling, R., Gylltoft, K. (2013). CDPM2: A damage-plasticity approach to modelling the failure of concrete. International Journal of Solids and Structures, 50(24):3805-3816.

[2] Wang, T., Hsu, T. (2001). Nonlinear finite element analysis of concrete structures using new constitutive models. Computers \& structures, 79:27812791.

[3] William, K.J., Warnke, E.P. (1975). Constitutive model for the triaxial behavior of concrete. In: Proceedings of the International Assoc. for Bridge and Structural Engineering, 19:1-30. 
[4] Trần Thế Truyền, Nguyễn Viết Trung (2011). Các mô hình ứng xử của bê tông, đánh giá mô hình tối ưu dùng trong mô phỏng số các kết cấu bê tông. Technical report, Trường Đại học Giao Thông Vận Tải.

[5] Lê Nhựt Trường (2013). Triển khai mô hình vật liệu microplane M4L cho phân tích phi tuyến kết cấu bê tông trong phần mềm ANSYS. Luận văn thạc sĩ, Trường Đại học Bách Khoa thành phố Hồ Chí Minh.

[6] Zdenek, P. B. and Pere, C. P. (1988). Microplane Model for Brittle-Plastic Material. I: Theory. Journal of Engineering Mechanics, 114(10):1672-1688.

[7] Bažant, Z. P., Caner, F. C., Carol, I., Adley, M. D., Akers, S. A. (2000). Microplane Model M4 for Concrete. I: Formulation with Work-Conjugate Deviatoric Stress. Journal of Engineering Mechanics, 126(9):944-953.

[8] Caner, F. C., Bažant, Z. P. (2012). Microplane Model M7 for Plain Concrete. I: formulation. Journal of Engineering Mechanics, 139(12):1714-1723.

[9] Pirker, M. (2020). Non-Linear Seismic Analysis of a Concrete Gravity Dam Using a Microplane Material Model. Final project for Master's degree, Graz University of Technology.

[10] Zreid, I., Kaliske, M. (2014). Regularization of Microplane Damage Models Using an Implicit Gradient Enhancement. International Journal of Solids and Structures, 51(19-20):3480-3489.

[11] Zreid, I., Kaliske, M. (2016). Implicit Gradient Formulation for Microplane Drucker-Prager Plasticity. International Journal of Plasticity, 83:252-272.

[12] Zreid, I., Kaliske, M. (2018). Gradient Enhanced Plasticity-Damage Microplane Model for Concrete. Computational Mechanics, 62:1239-1257.

[13] Nguyen, V. T., Li, J., Caner, F. C., (2013). Microplane Constitutive Model M4L for Concrete. I: Theory. Computers \& Structures, 128: 219-229.

[14] Cao, N. T., Pansuk, W., Torres, L. (2015). Flexural behavior of fire-Damaged RC slabs repaired with near-Surface mounted (NSM) carbon fiber reinforced polymer (CFRP) rods. Journal of Advanced Concrete Technology, 13(1):15-29.

[15] ACI 318-08 (2008). Building Code Requirements for Structural Concrete and Commentary. American Concrete Institute, p.345.

[16] Release 2020 R1 (2020) Material Reference. ANSYS Inc, pp.116-125.

[17] Feenstra, P. H., Borst, R. D. (1996). A Composite Plasticity Model for Concrete. International Journal of Solids and Structures, 33(5):707-730.

[18] Jiang, H., Zhao, J. (2015). Calibration of the Continuous Surface Cap Model for Concrete. Finite Elements in Analysis and Design, 97:1-19.

[19] Nguyen, D. G., Houlsby, G. T. (2008). A Coupled Damage-Plasticity Model for Concrete Based on Thermodynamic Principles: Part I: Model Formulation and Parameter Identification. International Journal for Numerical and Analytical Methods in Geomechanics, 32(4):353-389.

[20] Pham, D. D., Nguyen, P. C., Nguyen, D. L., Le, H. A. (2020). Simulation of Concrete-filled Steel Box Column. In: Reddy J., Wang C., Luong V., Le A. (eds) ICSCEA 2019. Lecture Notes in Civil Engineering, Vol 80. ICSCEA 2019. Springer, Singapore.

[21] Release 2020R1 (2020) ANSYS Mechanical APDL Theory Reference. ANSYS Inc. 